\title{
STIEFEL-WHITNEY NUMBERS OF QUATERNIONIC AND RELATED MANIFOLDS
}

\author{
BY
}

E. E. FLOYD

\begin{abstract}
There is considered the image of the symplectic cobordism ring $\Omega_{*}^{S p}$ in the unoriented cobordism ring $N_{*}$. A polynomial subalgebra of $N_{*}$ is exhibited, with all generators in dimensions divisible by 16 , such that the image is contained in the polynomial subalgebra. The methods combine the $K$-theory characteristic numbers as used by Stong with the use of the Landweber-Novikov ring.
\end{abstract}

Let $M$ be a closed smooth manifold of dimension $k$. For $n$ large, $M$ can be embedded in $S^{4 n+k}$ in essentially only one way. Let $\nu$ denote the normal bundle of the embedding. We say that $M$ is quaternionic if $v$ has the structure of a quaternionic vector bundle. Stong [11] proved that in dimensions $k<24$ all StiefelWhitney numbers of a quaternionic manifold $M^{k}$ vanish. In his thesis, David Segal [8] extended this to dimensions $k<32$. He also showed the existence of a quaternionic $M^{32}$ unorientedly cobordant to $[R P(2)]^{16}$.

To put the situation more generally, there is the unoriented cobordism ring $N_{*}=\sum N_{k}$, and there is a quaternionic cobordism group $\Omega_{*}^{S p}=\sum \Omega_{k}^{S p}$ [10]. There is a natural homomorphism $\Omega_{*}^{S p} \rightarrow N_{*}$, and $\operatorname{Im}\left(\Omega_{*}^{S p} \rightarrow N_{*}\right)$ is the subalgebra of $N_{*}$ consisting of all cobordism classes represented by quaternionic manifolds $M$. I am not able to compute this image, but I do give an upper bound to the image and now turn to an outline of the method. In their more widely ranging studies of symplectic cobordism, both Porter [14] and Segal have recently obtained very closely related results.

Let $A^{\prime}$ denote a graded vector space over $Z_{2}$ with basis consisting of all $s^{R}$, where $R$ ranges over all sequences $R=\left(r_{1}, r_{2}, \ldots\right)$ of nonnegative integers with $\sum r_{k}<\infty$ and where $\operatorname{deg} s^{R}=\sum k r_{k}$. Landweber [5] and Novikov [7] put a Hopf algebra structure on $A^{\prime}$. The product can be defined as composition of operators. Namely each $s^{R}$ acts on any polynomial algebra $Z_{2}\left[x_{1}, \ldots, x_{m}\right]$ by

$$
\begin{aligned}
s^{0}\left(x_{i}\right) & =x_{i}, \\
s^{\Delta_{k}}\left(x_{i}\right) & =x_{i}^{k+1} \quad \text { where } \quad \Delta_{k}=(0, \ldots, 0,1,0, \ldots), \\
s^{R}\left(x_{i}\right) & =0 \quad \text { if } R \neq 0 \text { and } R \neq \Delta_{k}, \\
s^{R}(y z) & =\sum_{R_{1}+R_{2}=R} s^{R_{1}}(y) s^{R_{2}}(z) .
\end{aligned}
$$

Received by the editors March 26, 1970.

AMS 1968 subject classifications. Primary 5710, 5732; Secondary 5730.

Key words and phrases. Quaternionic manifolds, symplectic cobordism, unoriented cobordism, Stiefel-Whitney numbers, $K$-theory characteristic numbers.

Copyright (C) 1971, American Mathematical Society 
The mod 2 Steenrod algebra $\mathscr{S}$ can be considered as a subalgebra of $A^{\prime}$ by identifying $S q^{k}$ with $s^{k \Delta_{1}}$. There is also the subalgebra $T$ of $A^{\prime}$ generated by $s^{\Delta_{2}}$ and all the $S q^{k}$. As always, $A^{\prime} / T^{+} \cdot A^{\prime}$ has a natural coproduct where $T^{+}$consists of all sums of elements of $T$ of positive degree. Its dual algebra is a polynomial algebra with generators in each dimension $2^{k}(k>1)$, each $2(2 k-1)$ where $k \neq 2^{j}$, and each $2 k$ with $k \neq 2^{j}$. More specifically, we show in $\S 4$ that $A^{\prime}$ is a free left $T$-module with basis elements $s^{R}$ for all $R$ with $r_{2^{j}-1}=0, r_{2 k-1}=0 \bmod 2$ for $k \neq 2^{j}, r_{2^{k}}=0 \bmod 2$.

Now let $M O$ denote the Thom spectrum of the orthogonal group, so that $\pi_{k}(M O)$ is the unoriented cobordism group $N_{k}$. There is the pairing

$$
\tilde{H}^{*}\left(M O ; Z_{2}\right) \otimes N_{*} \rightarrow Z_{2}
$$

sending $s^{R} \otimes[M]$ into the normal characteristic number $\bmod 2 s^{R}[M]$. We may identify $\tilde{H}^{*}\left(M O ; Z_{2}\right)$ with $A^{\prime}$ and obtain $A_{k}^{\prime} \otimes N_{k} \rightarrow Z_{2}$. According to Thom [12],

$$
\left(A^{\prime} / \mathscr{S}^{+} \cdot A^{\prime}\right)_{k} \otimes N_{k} \rightarrow Z_{2}
$$

is a dual pairing.

Define $P_{k} \subset N_{k}$ to be all cobordism classes $[M]$ of $N_{k}$ such that $\left(s^{\Delta_{2} S^{R}}\right)[M]=0$, for all $R$ of degree $k-2$. Then $P=\sum P_{k}$ is a subalgebra of $N_{*}$ and $P_{k}$ is the dual group of $\left(A^{\prime} / T^{+} A^{\prime}\right)_{k}$. In $\S 5$ we show that we can select a basis $\left[M^{2}\right],\left[M^{4}\right],\left[M^{5}\right], \ldots$ for the polynomial algebra $N_{*}$ such that $P$ is the subalgebra generated by all $\left[M^{2 k-1}\right]^{2}$ for $k \neq 2^{j},\left[M^{2^{k}}\right]^{2},\left[M^{2 k}\right]$ for $k \neq 2^{j}$.

Similarly define $U$ to be the subalgebra of $A^{\prime}$ generated by $s^{\Delta_{2}}, s^{2 \Delta_{2}}$ and all $S q^{k}$. In $\S 4$ the structure of $A^{\prime} / U^{+} A^{\prime}$ is computed. In $\S 5$ we let $Q_{k} \subset N_{k}$ be all [M] with

$$
\begin{aligned}
& \left(s^{\Delta_{2} S^{R}}\right)[M]=0, \quad \text { degree } R=k-2, \\
& \left(s^{2 \Delta_{2} S^{R}}\right)[M]=0, \quad \text { degree } R=k-4 .
\end{aligned}
$$

We show that $Q=\sum Q_{k}$ is given by $Q=P^{2}$.

Consider now the spectrum $M S p$ coming from the symplectic group. Then $\Omega_{*}^{S p}=\pi_{*}(M S p)$. There is the pairing

$$
\tilde{H} *\left(M S p ; Z_{2}\right) \otimes \Omega_{*}^{S p} \rightarrow Z_{2}
$$

We can regard $\tilde{H}^{*}\left(M S p ; Z_{2}\right)$ as another copy of the Hopf algebra $A^{\prime}$, with generators ' $S$. The two key remarks of $\S 3$ which make possible the use of $A^{\prime}$ are the following: if $[M] \in \Omega_{4 k}^{S p}$ then

$$
\left({ }^{\prime} S^{\Delta_{2} \cdot} \cdot S^{R}\right)[M]=0 \bmod 2, \quad R \text { of degree } k-2 ;
$$

if $[M] \in \Omega_{8 k}^{S p}$ then

$$
\left({ }^{2 \Delta_{2}} \cdot{ }^{\prime} S^{R}\right)[M]=0 \bmod 2, \quad \text { degree } R=k-4 .
$$

These assertions are proved by a very standard use of $K$-theory, in particular the Adams operations. $\S \S 2$ and 3 are largely devoted to a proof of these remarks. 
The homomorphism $\Omega_{*}^{S p} \rightarrow N_{*}$ comes from a map of spectra $g: M S p \rightarrow M O$. We have

$$
g^{*}\left(s^{4 R}\right)={ }^{\prime} S^{R}, \quad g^{*}\left(s^{R^{\prime}}\right)=0 \quad \text { if } R^{\prime} \neq 4 R .
$$

Hence if $[M] \in \operatorname{Im}\left(\Omega_{*}^{S p} \rightarrow N_{*}\right)$ then

$$
\begin{aligned}
\left(s^{4 \Delta_{2}} \cdot s^{R}\right)[M] & =0, & & \left(s^{8 \Delta_{2}} \cdot s^{R}\right)[M]=0, \\
s^{R^{\prime}}[M] & =0, & & R^{\prime} \neq 4 R .
\end{aligned}
$$

It follows without difficulty that

$$
\operatorname{Im}\left(\Omega_{*}^{S p} \rightarrow N_{*}\right) \subset Q^{4}=P^{8} .
$$

That is, there is a basis $\left[M^{2}\right],\left[M^{4}\right],\left[M^{5}\right], \cdots$ for $N_{*}$ such that $\operatorname{Im}\left(\Omega_{*}^{S p} \rightarrow N_{*}\right)$ is contained in the polynomial subalgebra generated by

$$
\left[M^{2}\right]^{16},\left[M^{4}\right]^{16},\left[M^{5}\right]^{16},\left[M^{6}\right]^{8}, \ldots
$$

Here $M^{2^{k}}$ and $M^{2 l-1}$ for $l \neq 2^{j}$ occur to the power $16, M^{2 k}$ for $k \neq 2^{j}$ to the power 8. This is one of the two main theorems of the paper.

While about it, it is easy to prove a similar assertion for another natural subalgebra $E$ of $N_{*}$. Let $E_{2 k}$ consist of all cobordism classes $N_{2 k}$ which are represented by weakly almost complex manifolds $M$ for which all Chern numbers

$$
c_{2 r+1} c_{i_{1}} \cdots c_{i_{p}}[M]
$$

vanish. We prove that $E \subset P^{4}$ so that $E$ is contained in the subalgebra generated by

$$
\left[M^{2}\right]^{8}, \quad\left[M^{4}\right]^{8}, \quad\left[M^{5}\right]^{8}, \quad\left[M^{6}\right]^{4}, \quad \ldots
$$

This is the other principal theorem of the paper. We conjecture that $E=P^{4}$; at least as in $\S 1$ one can construct a few nonzero elements of $E$. In fact, in a later paper we hope to prove $E=P^{4}$ using methods similar to those of Stong [11].

1. A few examples. The purpose of this section is to prove the following.

THEOREM. There is a closed smooth manifold $M^{16 n}$, unorientedly cobordant to $[R P(2 n)]^{8}$, whose stable tangent bundle

$$
\tau(M)-16 n \in \tilde{K} O(M)
$$

is of the form $2 y$ where $y \in \tilde{K} O(M)$.

Proof. Consider the complex Grassmann manifold

$$
M^{16 n}=C M(4 n, 2)
$$

consisting of all 2-dimensional vector subspaces $V$ of $C^{4 n+2}$. There is the bundle $\xi$ associating with each $V \in M$ all $x \in V$, the trivial bundle $4 n+2$ associating with $V$ 
all $x \in C^{4 n+2}$, and $\xi^{\perp}$ associating with $V$ all $x \in V^{\perp}$ where $V^{\perp}$ is the orthogonal complement of $V$ in $C^{4 n+2}$. The tangent bundle to $M$ is [4]

$$
\begin{aligned}
\tau(M) & =\operatorname{Hom}_{C}\left(\xi, \xi^{\perp}\right) \\
& =\operatorname{Hom}_{C}(\xi, 4 n+2)-\operatorname{Hom}_{C}(\xi, \xi) \\
& =(4 n+2) \xi-\operatorname{Hom}_{C}(\xi, \xi) .
\end{aligned}
$$

There is the operator $\alpha: \operatorname{Hom}_{C}(\xi, \xi) \rightarrow \operatorname{Hom}_{C}(\xi, \xi)$ where $\alpha(\varphi)$ is the adjoint $\varphi^{*}$ of $\varphi$. Clearly $\alpha$ is a conjugate linear involution. Let

$$
A_{+}=\{\varphi: \alpha(\varphi)=\varphi\}, \quad A_{-}=\{\varphi: \alpha(\varphi)=-\varphi\} .
$$

Then

$$
\operatorname{Hom}_{C}(\xi, \xi)=A_{+}+A_{-}
$$

and multiplication by $i$ is a real isomorphism between $A_{+}$and $A_{-}$. Hence $\operatorname{Hom}_{C}(\xi, \xi)$ is of the form $2 x$ in $\tilde{K} O(M)$, and $\tau(M)-16 n=2 y$ for some $y \in \tilde{K} O(M)$.

The equation $[C M(4 n, 2)]=[R P(2 n)]^{8}$ follows readily from the equations $[C M(n, k)]=[R M(n, k)]^{2},[R M(2 n, 2 k)]=[C M(n, k)]^{2}$. Here $C M(n, k)$ consists of all $k$-dimensional complex vector subspaces of $C^{n-k}$ and $R M(n, k)$ of all real $k$-dimensional real subspaces of $R^{n-k}$. Each of these follows from the theorem [1]: Let $T: M \rightarrow M$ be a smooth involution on the closed smooth manifold $M^{2 n}$, with fixed point set $F$ of dimension $n$; if the normal bundle to $F$ has the same StiefelWhitney classes as the tangent bundle of $F$, then $[M]=[F]^{2}$.

Consider $R^{2 n+2 k}=C^{n+k}$, and let $M=R M(2 n, 2 k)$. There is $T: M \rightarrow M$ defined by $T(V)=I(V)$ where $I$ is multiplication by $i$. The fixed point set $F$ is clearly $C M(n, k)$. The inclusion $i: F \subset M$ induces $i^{*} \tau(M)$ and

$$
i^{*} \tau(M)=\operatorname{Hom}_{R}\left(i^{*} \xi, i^{*} \xi^{\perp}\right)
$$

For $V \in F$, we have $V$ is a complex vector space and

$$
\operatorname{Hom}_{R}\left(V, V^{\perp}\right)=\operatorname{Hom}_{C}\left(V, V^{\perp}\right)+\operatorname{Hom}_{C}^{\prime}\left(V, V^{\perp}\right)
$$

where the second term denotes all conjugate linear homomorphisms. Now Hom $_{C}\left(i^{*} \xi, i^{*} \xi^{\perp}\right)$ is the tangent bundle $\tau(F)$, hence the normal bundle $\eta$ to $F$ in $M$ is

$$
\eta=\operatorname{Hom}_{C}^{\prime}\left(i^{*} \xi, i^{*} \xi^{\perp}\right)
$$

Hence

$$
\xi(F)=\overline{i^{*}(\xi)} \otimes_{C} i^{*} \xi^{\perp}, \quad \eta=\overline{i^{*} \xi} \otimes_{C} \overline{i^{*} \xi^{\perp}} .
$$

These two bundles have the same Chern classes reduced mod 2, hence they have the same Stiefel-Whitney classes. The second equation follows. The first follows readily from [1, pp. 64-65]. 
It is possible to put the above construction a little more generally, but I do not know how to take advantage of the added generality. Namely suppose $M$ is a closed smooth manifold whose stable tangent bundle is of the form $2 y$ for some $y \in \tilde{K} O(M)$. Let $\xi$ be a smooth complex bundle over $M$ and let $C M(2 \xi, 2)$ denote the space of all two-dimensional vector subspaces of fibers of $2 \xi$. Then $M^{\prime}$ $=C M(2 \xi, 2)$ has stable tangent bundle of the form $2 z$ for some $z \in \tilde{K} O\left(M^{\prime}\right)$.

Note that we can make $M^{16 n}$ weakly almost complex by assigning to its stable tangent bundle the complex structure of the complexification of $y$. In this complex structure we have $c_{2 r+1}\left(M^{16 n}\right)=0$ since on the one hand $c_{2 r+1}\left(M^{16 n}\right)$ is torsion and on the other hand $H^{*}\left(M^{16 n}\right)$ has no torsion. Hence

$$
c_{2 r+1} c_{i_{1}} \cdots c_{i_{p}}\left[M^{16 n}\right]=0
$$

whether the Chern numbers are those of the tangent bundle or the normal bundle. That is, $[R P(2 n)]^{8} \in E_{16 n}$.

2. The spectra of Thom spaces. Let $\xi$ be the canonical complex line bundle over $B U(1)$, and let $\eta$ be the canonical quaternionic line bundle over $B S p(1)$. There is a map

$$
g: B U(1) \rightarrow B S p(1)
$$

with $g^{*}(\eta)=\xi+\xi$. Let $v=\eta-2$ in $K(B S p(1))$. Then $K(B S p(1))$ is the ring of formal power series $Z[[v]]$. We may take $H^{*}(B S p(1))$ as $Z[u]$ for $u \in H^{4}(B S p(1))$, and suppose

$$
\operatorname{ch} v=u+\text { higher order terms. }
$$

Also if $\psi^{2}$ is the Adams operation,

$$
g^{*} \psi^{2}(\eta-2)=\psi^{2}(\xi+\xi-2)=\xi^{2}+\xi^{2}-2=(\xi+\xi-2)^{2}+4(\xi+\bar{\xi}-2)
$$

so that $\psi^{2}(v)=4 v+v^{2}$. More generally we can identify $K^{*}(B S p(1))$ with $K^{*}(p t)[[v]]$.

Similarly we can consider $v^{\prime}=\eta-1$ as an element of $\tilde{K} O^{4}() \approx \tilde{K} S p($ ) and

$$
K O^{*}(B S p(1))=K O^{*}(p t)\left[\left[v^{\prime}\right]\right] \text {. }
$$

The natural homomorphism $K O^{*}(\mathrm{l}) \rightarrow K^{*}(\mathrm{l})$ maps $v^{\prime}$ into $v$ and $v^{\prime n}$ into $v^{n}$.

One can next check that

$$
\begin{aligned}
H^{*}(B S p(1) \times \cdots \times B S p(1)) & \approx Z\left[u_{1}, \cdots, u_{n}\right], \\
K O^{*}(B S p(1) \times \cdots \times B S p(1)) & \approx K O^{*}(p t)\left[\left[v_{1}^{\prime}, \ldots, v_{n}^{\prime}\right]\right], \\
K^{*}(B S p(1) \times \cdots \times B S p(1)) & \approx K^{*}(p t)\left[\left[v_{1}, \ldots, v_{n}\right]\right]
\end{aligned}
$$

where ch $v_{i}=u_{i}+$ higher order terms, $\psi^{2}\left(v_{i}\right)=4 v_{i}+v_{i}^{2}, K O^{*}() \rightarrow K^{*}()$ maps $v_{i}^{\prime}$ into $v_{i}$.

One can use Dold's theorem [2] on the natural map

$$
B S p(1) \times \cdots \times B S p(1) \rightarrow B S p(n)
$$


to check that $H^{*}(B S p(n)), K O^{*}(B S p(n)), K^{*}(B S p(n))$ can be identified with the symmetric terms of

$$
Z\left[u_{1}, \ldots, u_{n}\right], \quad K O^{*}(p t)\left[\left[v_{1}^{\prime}, \ldots, v_{n}^{\prime}\right]\right], \quad K^{*}(p t)\left[\left[v_{1}, \ldots, v_{n}\right]\right]
$$

respectively.

Finally we identify $M S p(n)$ with $B S p(n) / B S p(n-1)$ to obtain an exact sequence

$$
\cdots \longrightarrow h^{*}(M S p(n)) \longrightarrow h^{*}(B S p(n)) \stackrel{f^{*}}{\longrightarrow} h^{*}(B S p(n-1)) \longrightarrow \cdots
$$

for $h=H, K O$ or $K$. In each case $f^{*}$ is an epimorphism with kernel the ideal generated by $u_{1} \cdots u_{n}, v_{1}^{\prime} \cdots v_{n}^{\prime}, v_{1} \cdots v_{n}$, respectively. Hence we identify $\tilde{H}^{*}(M S p)$ with the ideal of symmetric polynomials of $Z\left[u_{1}, \ldots, u_{n}\right]$ generated by $u_{1} \cdots u_{n}, \tilde{K}(M S p(n))$ with the ideal of symmetric formal series in $Z\left[\left[v_{1}, \ldots, v_{n}\right]\right]$ generated by $v_{1} \cdots v_{n}$, and $\tilde{K} O^{*}(M S p(n))$ with the ideal of symmetric elements of $K O^{*}\left[\left[v_{1}^{\prime}, \ldots, v_{n}^{\prime}\right]\right]$ generated by $v_{1}^{\prime} \cdots v_{n}^{\prime}$.

There is the spectrum $M S p$ generated by the $M S p(n)$, and the groups $\tilde{H}^{*}(M S p)$, $\widetilde{K} O^{*}(M S p), \tilde{K}^{*}(M S p)$ defined as inverse limits.

For each sequence $R=\left(r_{1}, r_{2}, \ldots, r_{k}, \ldots\right)$ of nonnegative integers with $|R|=\sum r_{k}<\infty$, there is the element ' $S^{R}$ of $\tilde{H}^{*}(M S p)$ represented in $\tilde{H}^{*}(M S p(n))$ by

$$
{ }^{\prime} S^{R}=\sum u_{1}^{2} \cdots u_{r_{1}}^{2} u_{r_{1}+1}^{3} \cdots u_{r_{1}+r_{2}}^{3} \cdot u_{r_{1}+r_{2}+1}^{4} \cdots u_{|R|+1} \cdots u_{n}
$$

for $n$ large.

Define $\|R\|=\sum k r_{k}$, so that ' $S^{R} \in H^{4\|R\|}(M S p)$.

Hence we identify $\tilde{H}^{*}(M S p)$ with the free abelian group $A$ generated by all ' $S^{R}$ for all $R$. Similarly $\tilde{K}(M S p)$ contains a free abelian group $A$ generated by all $S^{R}$, where $S^{R}$ is represented in $\tilde{K}(M S p(n))$ by

$$
S^{R}=\sum v_{1}^{2} \cdots v_{r_{1}}^{2} v_{r_{1}+1}^{3} \cdots v_{r_{1}+r_{2}}^{3} \cdots v_{|R|+1} \cdots v_{n} .
$$

Moreover ch $S^{R}={ }^{\prime} S^{R}+$ higher order terms.

There is the quaternionic cobordism group $\Omega_{k}^{S p}[10]$, which may either be regarded as all bordism classes of closed smooth $k$-manifolds with given quaternionic structure on the stable normal bundle, or as all homotopy classes of maps

$$
f: S^{4 n+k} \rightarrow M S p(n), \quad n \text { large. }
$$

Given $[M] \in \Omega_{4 k}^{S p}$ represented by $f: S^{4 n+4 k} \rightarrow M S p(n)$ and given ' $S^{R}$, we get the integer

$$
' S^{R}[M]=\left\langle f^{*}{ }^{\prime} S^{R}, \sigma_{4 n+4 k}\right\rangle
$$

where $\sigma_{4 n+4 k}$ is the orientation class of $S^{4 n+4 k}$. Note that ' $S^{R}[M]=0$ unless $\|R\|$ $=k$. Similarly for each $S^{R} \in \tilde{K}(M S p)$, we get the integer

$$
S^{R}[M]=\left\langle\operatorname{ch} f^{*} S^{R}, \sigma_{4 n+4 k}\right\rangle,
$$

and $S^{R}[M]=0$ unless $\|R\| \leqq k$. If $\|R\|=k$, then $S^{R}[M]={ }^{\prime} S^{R}[M]$. 
The above completes the notation concerning the spectrum MSp. Note that an equivalent discussion holds for $M U$ except for the remarks about $K O^{*}$. Namely $\tilde{H}^{*}(M U)$ can be identified with the free abelian group $A$ generated by all ' $S^{R}$, where ' $S^{R}$ is represented in $H^{*}(M U(n))$ by

$$
{ }^{\prime} S^{R}=\sum x_{1}^{2} \cdots x_{r_{1}}^{2} x_{r_{1}+1}^{3} \cdots x_{r_{1}+r_{2}}^{3} \cdots x_{|R|+1} \cdots x_{n} .
$$

Similarly if $[M] \in \Omega_{2 k}^{U}$ is represented by $f: S^{2 n+2 k} \rightarrow M U(n)$, there are the integers ${ }^{\prime} S^{R}[M], S^{R}[M]$ defined as before.

Finally there is the spectrum $M O$. The cohomology group $\tilde{H}^{*}\left(M O ; Z_{2}\right)$ can be identified with the vector space over $Z_{2}$ with basis all $s^{R}$ where $s^{R}$ is represented in $\tilde{H}^{*}\left(M O(n) ; Z_{2}\right)$ by

$$
s^{R}=\sum z_{1}^{2} \cdots z_{r_{1}}^{2} z_{r_{1}+1}^{3} \cdots z_{r_{1}+r_{2}}^{3} \cdots z_{|R|+1} \cdots z_{n}
$$

For $[M] \in N_{k}$, there is the integer $\bmod 2$

$$
s^{R}[M]=\left\langle f^{*} s^{R}, \sigma_{n+k}^{\prime}\right\rangle .
$$

There are natural maps of spectra

$$
M S p \stackrel{g}{\longrightarrow} M U \stackrel{h}{\longrightarrow} M O .
$$

We assume the following facts:

(a) $g^{*}: \widetilde{K}(M U) \rightarrow \widetilde{K}(M S p)$ is an epimorphism,

(b) we have $h^{*}: \tilde{H}^{*}\left(M O ; Z_{2}\right) \rightarrow \tilde{H}^{*}\left(M U ; Z_{2}\right)$ given by $h^{*}\left(s^{2 R}\right)={ }^{\prime} S^{R} \bmod 2, h^{*}\left(s^{R}\right)$ $=0$ if $R \neq 2 R^{\prime}$,

(c) $g^{*}: \tilde{H}^{*}\left(M U ; Z_{2}\right) \rightarrow \tilde{H}^{*}\left(M S p ; Z_{2}\right)$ is given by $g^{*}\left({ }^{\prime} S^{2 R}\right)={ }^{\prime} S^{R} \bmod 2, g^{*}\left({ }^{\prime} S^{R}\right)$ $=0$ if $R \neq 2 R^{\prime}$.

3. Divisibility relations among characteristic numbers. Denote by $A$ a free abelian group with basis elements $S^{R}$, one for each sequence $R=\left(r_{1}, r_{2}, \ldots\right)$ of nonnegative integers with $|R|=\sum r_{k}<\infty$. Following Landweber [5] and Novikov [7], there is a multiplication defined on $A$. Each $S^{R}$ operates as a group homomorphism on any polynomial algebra $Z\left[x_{1}, \ldots, x_{n}\right]$ with given generators by

$$
\begin{aligned}
S^{0}\left(x_{i}\right) & =x_{i}, \\
S^{\Delta_{k}}\left(x_{i}\right) & =x_{i}^{k+1} \quad \text { where } \Delta_{k}=(0, \ldots, 0,1,0, \ldots), \\
S^{R}\left(x_{i}\right) & =0 \quad \text { if } R \neq 0 \text { and } R \neq \Delta_{k}, \\
S^{R}(y z) & =\sum_{R_{1}+R_{2}=R} S^{R_{1}}(y) \cdot S^{R_{2}}(z),
\end{aligned}
$$

for all $y, z \in Z\left[x_{1}, \ldots, x_{n}\right]$.

Given $R, R^{\prime}$ there exists a unique $T \in A$ with $S^{R} \circ S^{R^{\prime}}=T$ on all $Z\left[x_{1}, \ldots, x_{n}\right]$. In this fashion $A$ becomes an associative ring with unit. 
For each nonnegative integer $k$ there is also an operator

$$
S Q^{k}: Z\left[x_{1}, \ldots, x_{n}\right] \rightarrow Z\left[x_{1}, \ldots, x_{n}\right]
$$

defined by

$$
\begin{aligned}
S Q^{0}\left(x_{i}\right) & =x_{i}, \quad S Q^{1}\left(x_{i}\right)=x_{i}^{2}, \\
S Q^{k}\left(x_{i}\right) & =0 \text { for } k>1, \\
S Q^{k}(y z) & =\sum_{i+j=k} S Q^{t}(y) \cdot S Q^{y}(z) .
\end{aligned}
$$

As operators, we have $S Q^{k}=S^{(k, 0,0, \ldots)}$ and thus we identify $S Q^{k}$ with $S^{(k, 0, \ldots)} \in A$. There are the formulas

$$
S^{R}\left(x_{i}^{m}\right)=\frac{m !}{r_{1} ! r_{2} ! \cdots(m-|R|) !} x_{i}^{m+\|R\|}, \quad S Q^{k}\left(x_{i}^{m}\right)=\left(\begin{array}{l}
m \\
k
\end{array}\right) x_{i}^{m+k} .
$$

An element $T \in A$ is uniquely determined by its operator value $T\left(x_{1} x_{2} \cdots x_{n}\right)$ in $Z\left[x_{1}, \ldots, x_{n}\right]$ for $n$ large. Thus

$$
\begin{gathered}
S^{\Delta_{2}}\left(x_{1} \cdots x_{n}\right)=\sum x_{1}^{3} x_{2} \cdots x_{n}, \\
\left(S Q^{1} \cdot S Q^{1}-2 S Q^{2}\right)\left(x_{1} \cdots x_{n}\right)=2 \sum x_{1}^{3} x_{2} \cdots x_{n},
\end{gathered}
$$

and hence

$$
S^{\Delta_{2}}=\frac{1}{2} S Q^{1} \cdot S Q^{1}-S Q^{2}
$$

Similarly

$$
\begin{gathered}
S^{2 \Delta_{2}}\left(x_{1} \cdots x_{n}\right)=\sum x_{1}^{3} x_{2}^{3} x_{3} \cdots x_{n}, \\
\left(S Q^{2} \cdot S Q^{2}-S Q^{3} \cdot S Q^{1}-S Q^{1} \cdot S Q^{3}+2 S Q^{4}\right)\left(x_{1} \cdots x_{n}\right)=4 \sum x_{1}^{3} x_{2}^{3} x_{3} \cdots x_{n},
\end{gathered}
$$

and hence

$$
S^{2 \Delta_{2}}=\frac{1}{4}\left(S Q^{2} \cdot S Q^{2}-S Q^{3} \cdot S Q^{1}-S Q^{1} \cdot S Q^{3}+2 S Q^{4}\right) .
$$

We now turn to the pairing

$$
A \otimes \Omega_{*}^{S p} \rightarrow Z, \quad S^{R} \otimes[M] \rightarrow S^{R}[M]
$$

of $\S 2$ where $S^{R} \in \widetilde{K}(M S p)$. Let $f: S^{4 n+4 k} \rightarrow M S p(n)$ and let $S^{R} \in \tilde{K}(M S p(n))$ where $r=\|R\| \leqq k$. Then

$$
\begin{aligned}
S^{R} & =\sum v_{1}^{2} \cdots v_{r_{1}+1}^{3} \cdots v_{|R|+1} \cdots v_{n}, \\
\psi^{2} S^{R} & =\sum\left(4 v_{1}+v_{1}^{2}\right)^{2} \cdots\left(4 v_{r_{1}+1}+v_{r_{1}+1}^{2}\right)^{3} \cdots \\
& =4^{n+r} S^{R}+4^{n+r-1}\left(S Q^{1} \cdot S^{R}\right)+\cdots+4^{n-k+2 r}\left(S Q^{k-r} \cdot S^{R}\right)+\cdots
\end{aligned}
$$


Also

$$
\begin{aligned}
\left(\psi^{2} S^{R}\right)[M] & =\left\langle\operatorname{ch} f^{*} \psi^{2} S^{R}, \sigma_{4 n+4 k}\right\rangle \\
& =4^{n+k}\left\langle\operatorname{ch} f^{*} S^{R}, \sigma_{4 n+4 k}\right\rangle=4^{n+k} S^{R}[M]
\end{aligned}
$$

Combining the two equations and dividing by $4^{n-k+2 r}$, we get

$$
4^{2 k-2 r} S^{R}[M]=4^{k-r} S^{R}[M]+\cdots+4\left(S Q^{k-r-1} \cdot S^{R}\right)[M]+\left(S Q^{k-r} \cdot S^{R}\right)[M] .
$$

Recall here that $[M] \in \Omega_{4 k}^{S p}$ and that $\|R\|=r \leqq k$.

(3.4) Corollary. For each $[M] \in \Omega_{4 k}^{S p}$ and each $S^{R}$ of degree $r<k$, we have $\left(S Q^{k-r} \cdot S^{R}\right)[M]=0 \bmod 4$.

(3.5) If $[M] \in \Omega_{8 k}^{S p}$ and if $S^{R} \in \tilde{K}(M S p)$ is of degree $r<2 k$, then $\left(S Q^{2 k-r} \cdot S^{R}\right)[M]$ $=0 \bmod 8$.

Proof. Consider $M S p(2 n)$, and consider first $R$ with $\|R\|$ odd. Since $\|R\|$ is odd, in $\widetilde{K} O^{4}(M S p(2 n))$ there is the element

$$
z=\sum v_{1}^{\prime 2} \cdots v_{r_{1}+1}^{\prime 3} \cdots v_{|R|+1}^{\prime} \cdots v_{2 n}^{\prime}
$$

and $\tilde{K} O^{*}() \rightarrow \tilde{K}()$ maps $z$ into $S^{R}$. Since $\tilde{K} O^{4}() \approx \tilde{K} S p($ ), we may as well suppose that $S^{R}$ is a quaternionic bundle. Then

$$
S^{R}\left[M^{8 k}\right]=\left\langle\operatorname{ch} f^{*}\left(S^{R}\right), \sigma_{8 n+8 k}\right\rangle
$$

and since we can consider $f^{*}\left(S^{R}\right) \in \tilde{K} S p\left(S^{8 n+8 k}\right)$, we get $S^{R}[M]=0 \bmod 2$. In particular if $\|R\|=2 k-1$, (3.3) then gives $12 S^{R}\left[M^{8 k}\right]=\left(S Q^{1} \cdot S^{R}\right)\left[M^{8 k}\right]$ and hence

$$
\left(S Q^{1} \cdot S^{R}\right)[M]=0 \bmod 8, \quad\|R\|=2 k-1 .
$$

The general case now follows from (3.3), for $\left(S Q^{2 k-r-1} \cdot S^{R}\right)[M]=0 \bmod 2$, by the case already treated.

We can now convert (3.4) and (3.5) into remarks about cohomology characteristic numbers.

(3.6) For all $\left[M^{4 k}\right] \in \Omega_{4 k}^{S p}$ and all ' $S^{R} \in H^{4 k-8}(M S p)$ we have

$$
\left({ }^{\prime} S^{\Delta_{2} \cdot} \cdot S^{R}\right)\left[M^{4 k}\right]=0 \bmod 2 .
$$

Here the product is that of $A=\tilde{H}^{*}(M S p)$.

Proof. We have

$$
\begin{aligned}
\left({ }^{\prime} S^{\Delta_{2}} \cdot{ }^{\prime} S^{R}\right)[M] & =\left(S^{\Delta_{2}} \cdot S^{R}\right)[M] \\
& =\left(\left(\frac{1}{2} S Q^{1} \cdot S Q^{1}-S Q^{2}\right) \cdot S^{R}\right)[M]=0 \bmod 2
\end{aligned}
$$

by (3.4).

(3.7) For all $\left[M^{8 k}\right] \in \Omega_{8 k}^{S p}$ and all $S^{R} \in \tilde{H}^{8 k-16}(M S p)$ we have

$$
\left({ }^{\prime} S^{2 \Delta_{2}} \cdot S^{R}\right)\left[M^{8 k}\right]=0 \bmod 2 .
$$


Proof. We have

$$
\begin{aligned}
& \left({ }^{2} S^{2 \Delta_{2} \cdot} \cdot S^{R}\right)[M]=\left(S^{2 \Delta_{2}} \cdot S^{R}\right)[M] \\
& =\left(\frac{1}{4}\left(S Q^{2} \cdot S Q^{2}-S Q^{3} \cdot S Q^{1}-S Q^{1} \cdot S Q^{3}+2 S Q^{4}\right) \cdot S^{R}\right)[M] \\
& =0 \bmod 2
\end{aligned}
$$

by (3.5).

We shall next see that (3.6) applies to a considerably more general class of manifolds $M$.

Consider $\tilde{H}^{*}(M U(2 n))$ as the ideal of $H^{*}(B U(2 n))$ generated by $c_{2 n}$. In particular, consider $\tilde{H}^{*}(M U(2 n))$ as a module over $H^{*}(B U(2 n))$. The natural map $g: M S p(n) \rightarrow M U(2 n)$ then has

$$
g^{*}: H^{*}(M U(2 n)) \rightarrow H^{*}(M S p(n))
$$

an epimorphism with kernel the submodule generated by $c_{1}, c_{3}, \ldots, c_{2 k+1}, \ldots$ Hence if

$$
g^{*}: \widetilde{K}(M U(2 n)) \rightarrow \widetilde{K}(M S p(n))
$$

has $g^{*}(T)=0$, then $\operatorname{ch} T$ has every term in the submodule generated by $c_{1}, c_{3}, \ldots, c_{2 k+1}, \ldots$

Consider now the pairing

$$
\tilde{K}(M U) \otimes \Omega_{4 k}^{U} \rightarrow Z, \quad T \otimes[M] \rightarrow T[M] .
$$

It follows readily from the above that if $g^{*}(T)=0$ and if every Chern number of $M^{4 k}$ involving any $c_{2 r+1}$ vanishes, then $T\left[M^{4 k}\right]=0$. Recall that

$$
g^{*}: \tilde{K}(M U) \rightarrow \widetilde{K}(M S p)
$$

is also an epimorphism. Given $S^{R} \in \tilde{K}(M S p)$, select $T \in \widetilde{K}(M U)$ with $g^{*}(T)=S^{R}$. Given $\left[M^{4 k}\right] \in \Omega_{4 k}^{U}$ such that every Chern number of $M$ involving any $c_{2 r+1}$ vanishes, then define $S^{R}\left[M^{4 k}\right]=T\left[M^{4 k}\right]$. Note that $S^{R}\left[M^{4 k}\right]$ is an integer.

We next see that (3.3) holds for this wider class of manifolds. Recall that $\Omega_{*}^{S p} \rightarrow \Omega_{*}^{U}$ maps $\Omega_{*}^{S p}$ into the subalgebra $W$ of $\Omega_{*}^{U}$ consisting of all $\left[M^{4 k}\right]$ such that every Chern number of $\left[M^{4 k}\right]$ involving any $c_{2 r+1}$ vanishes. Moreover $\Omega_{*}^{S p} \rightarrow W$ is an isomorphism modulo 2-torsion groups. Hence given $\left[M^{4 k}\right] \in W$ there is $2^{s}$ with $2^{s}\left[M^{4 k}\right] \in \operatorname{Im} \Omega_{*}^{S p}$. Then (3.3) holds for $2^{s} M^{4 k}$ and hence also for $M^{4 k}$. We can now easily obtain the following.

(3.8) Suppose that $\left[M^{4 k}\right] \in \Omega_{4 k}^{U}$ has all Chern numbers involving any $c_{2 r+1}$ vanishing. Then for every ' $S^{R}$ in $\tilde{H}^{*}(M S p)$ with $\|R\|=k$, the number ${ }^{\prime} S^{R}\left[M^{4 k}\right]$ is well defined and $\left({ }^{\prime} S^{\Delta_{2}} \cdot{ }^{\prime} S^{R}\right)\left[M^{4 k}\right]=0 \bmod 2,\|R\|=k-2$.

4. The mod 2 algebra $A \otimes Z_{2}$. We now set up the machinery to properly utilize (3.7) and (3.8). The starting point is the work of Landweber on the Landweber-Novikov algebra. 
Let $A^{\prime}$ be a vector space over $Z_{2}$ with basis consisting of all $s^{R}$ where $R$ ranges over all sequences $R=\left(r_{1}, r_{2}, \ldots\right)$ of nonnegative integers with $\sum r_{k}<\infty$. Every element of $A^{\prime}$ acts on any polynomial algebra $Z_{2}\left[x_{1}, \ldots, x_{n}\right]$ exactly as in $\S 3$. This determines an associative ring structure on $A^{\prime}$, and $A^{\prime}=A \otimes Z_{2}$. There are also operators

$$
S q^{k}: Z_{2}\left[x_{1}, \ldots, x_{n}\right] \rightarrow Z_{2}\left[x_{1}, \ldots, x_{n}\right]
$$

exactly as before, and we consider $S q^{k}=s^{(k, 0, \cdots)} \in A^{\prime}$. Moreover $A^{\prime}$ is a Hopf algebra with coproduct

$$
\psi\left(s^{R}\right)=\sum_{R_{1}+R_{2}=R} s^{R_{1}} \otimes s^{R_{2}}
$$

Denote by $\mathscr{S} \subset A^{\prime}$ the subalgebra generated by all the $S q^{k}, k \geqq 0$. Reasoning of the type of Milnor (see Landweber again) shows that $\mathscr{S}$ has as additive basis all $s^{R}$ for all $R=\left(r_{1}, r_{2}, \ldots\right)$ such that $r_{k}=0$ whenever $k$ is not of the form $2^{j}-1$.

We can now set the problem of this section. Denote by $T \subset A^{\prime}$ the subalgebra generated by $s^{\Delta_{2}}$ and by all $S q^{k}$; denote by $U \subset A^{\prime}$ the subalgebra generated by $s^{\Delta_{2}}$, by $s^{2 \Delta_{2}}$, and by all $S q^{k}$. Then $A^{\prime}$ is a left $T$-module and there is $A^{\prime} / T^{+} \cdot A^{\prime}$. Moreover $A^{\prime}$ is a left $U$-module and there is $A^{\prime} / U^{+} \cdot A^{\prime}$. In this section we determine the structure of $A^{\prime} / T^{+} \cdot A^{\prime}$ and $A^{\prime} / U^{+} \cdot A^{\prime}$.

Linearly order the sequences $R$ by

(i) $R<R^{\prime}$ if $\|R\|<\left\|R^{\prime}\right\|$,

(ii) $R<R^{\prime}$ if $\|R\|=\left\|R^{\prime}\right\|$ and if $r_{1}=r_{1}^{\prime}, \ldots, r_{k}=r_{k}^{\prime}, r_{k+1}>r_{k+1}^{\prime}$.

Note that this is a well-ordering, since for any $k$ there are only a finite number of $R$ with $\|R\|=k$.

For any $R, R^{\prime}$ we have

$$
\begin{aligned}
s^{R} s^{R^{\prime}}\left(x_{1} \cdots x_{m}\right) & =s^{R}\left(\sum x_{1}^{2} \cdots x_{r_{1}}^{2} x_{r_{1}+1}^{3} \cdots\right) \\
& =a\left(R, R^{\prime}\right) s^{R+R^{\prime}}+\sum_{T>R+R^{\prime}} a_{T} s^{T},
\end{aligned}
$$

where

$$
a\left(R, R^{\prime}\right)=\prod\left(\begin{array}{c}
r_{k}+r_{k}^{\prime} \\
r_{k}
\end{array}\right) \bmod 2
$$

Hence if $a\left(R, R^{\prime}\right)=1 \bmod 2$ then

$$
s^{R} S^{R^{\prime}}=s^{R+R^{\prime}}+\sum_{T>R+R^{\prime}} a_{T} s^{T}
$$

(4.1) Suppose that $x_{i}$ and $y_{j}$ are sequences of elements of $A^{\prime}$, where

$$
x_{i} y_{j}=s^{R_{i, j}}+\sum_{T>R_{i, j}} a_{T} s^{T} .
$$


Suppose for $i \neq k$ or for $j \neq l$ that $R_{i, j} \neq R_{k, l}$. The elements $x_{i}, y_{j}$ are then linearly independent in $A^{\prime}$.

The proof is quite clear. For in any set of elements $R_{i, j}$ there is at least one.

We need now a few computations (see Landweber). Let $s_{k}$ denote $s^{\Delta_{k}}$ and let $s_{k, k}$ denote $s^{2 \Delta_{k}}$.

$$
\begin{aligned}
\left(s_{2 p} s_{2 q+1}\right)\left(x_{1} x_{2} \cdots x_{n}\right) & =s_{2 p}\left(\sum x_{1}^{2 q+2} x_{2} \cdots x_{n}\right) \\
& =\sum x_{1}^{2 q+2} x_{2}^{2 p+1} \cdots x_{n}, \\
\left(s_{2 q+1} s_{2 p}\right)\left(x_{1} \cdots x_{n}\right) & =s_{2 q+1}\left(\sum x_{1}^{2 p+1} x_{2} \cdots x_{n}\right) \\
& =\sum x_{1}^{2 p+2 q+2} x_{2} \cdots x_{n}+\sum x_{1}^{2 p+1} x_{2}^{2 q+2} \cdots x_{n}
\end{aligned}
$$

and

$$
s_{2 p} s_{2 q+1}+s_{2 q+1} s_{2 p}=s_{2 p+2 q+1}
$$

Similarly

$$
s_{2 p} s_{2 p}=s_{4 p}
$$

so that $s_{2^{k+1}}=\left(s_{2}\right)^{2^{k}}$. Also

$$
s_{2 p+1} s_{2 q+1}=s_{2 q+1} s_{2 p+1}, \quad\left(s_{2 p+1}\right)^{2}=0 .
$$

Moreover for $k>1$,

$$
\begin{aligned}
\left(S q^{n} \cdot s_{k}\right)\left(x_{1} \cdots x_{m}\right) & =S q^{n}\left(\sum x_{1}^{k+1} x_{2} \cdots x_{n}\right) \\
& =\sum_{r}\left(\left(\begin{array}{c}
k+1 \\
r
\end{array}\right) \sum x_{1}^{k+r+1} x_{2}^{2} \cdots x_{n-r+1}^{2} x_{n-r+2} \cdots\right) \\
& =\sum\left(\begin{array}{c}
k+1 \\
r
\end{array}\right) s_{k+r} \cdot S q^{n-r}\left(x_{1} \cdots x_{m}\right)
\end{aligned}
$$

and

$$
S q^{n} s_{k}=\sum\left(\begin{array}{c}
k+1 \\
r
\end{array}\right) s_{k+r} \cdot S q^{n-r}, \quad k>1 .
$$

Recall that $T$ is generated by $s_{2}$ and the $S q^{k}$. Since $s_{1} \in \mathscr{S} \subset T$ and $s_{2} \in T$ we obtain inductively that $s_{2 k+1} \in T$ from $s_{2 k+1}=s_{2} s_{2 k-1}+s_{2 k-1} s_{2}$. Since $s_{2 j-1} \in \mathscr{S}$, the new information here is that $s_{2 k-1} \in T$ for $k \neq 2^{j}$. Also $s_{2^{k+1}} \in T$.

It follows from (4) that

$$
\begin{aligned}
S q^{n} \cdot S_{2}^{k}=S_{2}^{k} \cdot S q^{n}+s_{2^{k}+1} & \cdot S q^{n-1}+s_{2^{k+1}} \cdot S q^{n-2^{k}}+s_{2^{k+1}+1} \cdot S q^{n-2^{k}-1} \\
S q^{n} \cdot s_{2 k+1} & =\sum\left(\begin{array}{c}
2 k+2 \\
r
\end{array}\right) s_{2 k+r+1} \cdot S q^{n-r} \\
& =\sum n_{2 r} s_{2 k+2 r+1} \cdot S q^{n-2 r} .
\end{aligned}
$$

(4.2) $T$ has as additive basis all

$$
X=\left(s_{2}^{\varepsilon_{2}} s_{4}^{\varepsilon_{4}} \cdots s_{2^{k}}^{\varepsilon^{k}} \cdots\right)\left(s_{5}^{\varepsilon_{5}} s_{9}^{\varepsilon_{9}} S_{11}^{\varepsilon_{11}} \cdots\right) s^{\left(r_{1}, 0, r_{3}, 0,0,0, r_{7}, 0, \ldots\right)}
$$

where $\varepsilon_{k}=0$ or 1 and where all but a finite number of the parameters are zero. 
Proof. Letting

$$
\begin{aligned}
x_{i} & =\left(s_{2}^{\varepsilon_{2}} s_{4}^{\varepsilon_{4}} \cdots\right)\left(s_{5}^{\varepsilon_{5}} s_{9}^{\varepsilon_{9}} \cdots\right), \\
y_{j} & =s^{\left(r_{1}, 0, r_{3}, 0,0,0, r_{7}, \cdots\right)}, \\
R_{i, j} & =\left(r_{1}, \varepsilon_{2}, r_{3}, \varepsilon_{4}, \varepsilon_{5}, 0, r_{7}, \varepsilon_{8}, \ldots\right),
\end{aligned}
$$

we see that $x_{i} y_{j}=s^{R_{i, j}}+$ higher terms. Linear independence then follows from (4.1). That every element of $T$ can be written as a linear combination of elements in the form $X$ follows from (1), (2), (3), (5), (6).

(4.3) TheOREM. Consider $A^{\prime}$ as a T-module under left multiplication by $T$. Then $A^{\prime}$ is a free $T$-module with basis all elements $s^{R}$ for all $R$ with $r_{2^{j}-1}=0, r_{2^{k}}$ $=0 \bmod 2, r_{2 s-1}=0 \bmod 2$ for $s \neq 2^{j}$.

Proof. That $A^{\prime}$ is a free $T$-module follows from Milnor-Moore [6], but this is also self-contained. Let

$$
\begin{aligned}
x_{i} & =\left(s_{2}^{\varepsilon_{2}} s_{4}^{\varepsilon_{4}} \ldots\right)\left(s_{5}^{\varepsilon_{5}} s_{9}^{\varepsilon_{9}} \cdots\right) s^{\left(r_{1}, 0, r_{3}, 0, \ldots\right)} \\
y_{j} & =s^{\left(0,2 r_{2}, 0,2 r_{4}, 2 r_{5}, r_{6}, \ldots\right)} \\
R_{i, j} & =\left(r_{1}, 2 r_{2}+\varepsilon_{2}, r_{3}, 2 r_{4}+\varepsilon_{4}, 2 r_{5}+\varepsilon_{5}, r_{6}, \ldots\right) .
\end{aligned}
$$

Then $x_{i} y_{j}=s^{R_{i, j}}+$ larger terms. Hence the elements $y_{j}$ are linearly independent in $A^{\prime}$ considered as a $T$-module, using (4.1). Since every $R$ is of the form $R_{i, j}$ it is clear from induction that the elements $x_{i} y_{j}$ generate $A^{\prime}$ as a vector space, and hence the $y_{j}$ generate $A^{\prime}$ as a $T$-module. The theorem follows.

There is a ring homomorphism $\alpha: A^{\prime} \rightarrow A^{\prime}$ defined by

$$
\alpha\left(s^{2 R}\right)=s^{R}, \quad \alpha\left(s^{R^{\prime}}\right)=0 \quad \text { if } R^{\prime} \neq 2 R .
$$

For consider a polynomial algebra

$$
M=Z_{2}\left[x_{1}, \ldots, x_{k}, \ldots\right]
$$

and in it consider the subalgebra

$$
N=\left\{y^{2} \mid y \in M\right\}
$$

Now each $s^{R}$ acts on $M$ and $s^{2 R}\left(y^{2}\right)=\left(s^{R}(y)\right)^{2}, s^{R^{*}}\left(y^{2}\right)=0$ for $R^{\prime} \neq 2 R$. Thus if $R^{\prime} \neq 2 R$ then $s^{R^{\prime}}$ acts trivially on $N$, and there is the commutative diagram

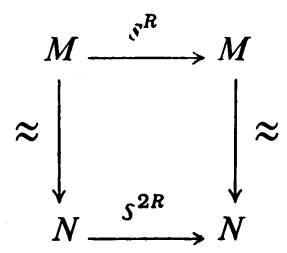


where $M \approx N$ is the squaring map. Thus we let $\alpha\left(s^{R^{\prime}}\right)$ be the unique $s^{R}$ with commutativity holding in

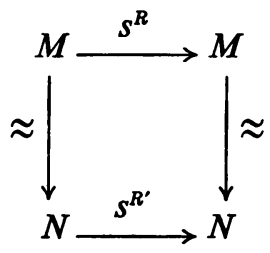

In particular, $\alpha\left(s_{2,2}\right)=s_{2}, \alpha\left(S q^{2 k}\right)=S q^{k}, \alpha\left(s_{2}\right)=0, \alpha\left(S q^{2 k+1}\right)=0$, and $\alpha(U)=T$ where $U$ is the subalgebra generated by $s_{2}, s_{2,2}$ and the $S q^{k}$. It follows that $\alpha$ maps $A^{\prime} / U^{+} \cdot A^{\prime}$ onto $A^{\prime} / T^{+} \cdot A^{\prime}$.

(4.4) THEOREM. The homomorphism $\alpha: A^{\prime} \rightarrow A^{\prime}$ induces an isomorphism

$$
A^{\prime} / U^{+} \cdot A^{\prime} \approx A^{\prime} / T^{+} \cdot A^{\prime}
$$

of vector spaces.

Proof. We need the following multiplication formulas.

(1) $s_{2,2} s_{4 k-2}+s_{4 k-2} s_{2,2}=s_{4 k+2}$,

(2) $s_{2,2} s_{2,2}+s_{2,2} s_{4}+s_{2} s_{2,2,2}=s_{4,4}$,

(3) $\left(s_{2^{k}}, 2^{k}\right)^{2}=s_{2^{k+1}}, 2^{k+1}, k \geqq 2$,

(4) $s_{2 k-3,2 k-3} s_{4,4}+s_{4} s_{2 k+1,2 k-3}+s_{4,4} s_{2 k-3,2 k-3}+\left({ }^{2 k-2}\right) s_{2 k+5,2 k-3}=s_{2 k+1,2 k+1}$.

It follows from (1) that $s_{4 k+2} \in U$. Hence also $s_{8 k+4}=\left(s_{4 k+2}\right)^{2} \in U, \ldots$ and all

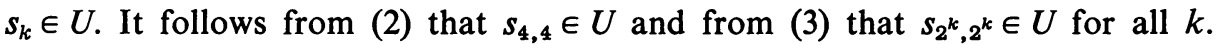
Finally (4) implies that $s_{2 k+1,2 k+1} \in U$ for all $k$.

To prove the theorem, we have only to check that the elements $s^{R}$ for which $r_{2^{j}-1}=0, r_{2 s-1}=0 \bmod 4$ for $s \neq 2^{j}, r_{2^{k}}=0 \bmod 4, r_{2 t}=0 \bmod 2$ for $t \neq 2^{j}$ generate $A^{\prime}$ as a $U$-module. For clearly the epimorphism $A^{\prime} / U^{+} \cdot A^{\prime} \rightarrow A^{\prime} / T^{+} \cdot A^{\prime}$ maps these elements into a basis.

Fix $m$ and consider all $R$ with $\|R\|=m$. The largest such $s^{R}$ is $s^{\Delta_{m}}$ and $s^{\Delta_{m}} \in U^{+}$. Fix $R$ and assume that for every $R^{\prime}>R$ with $\left\|R^{\prime}\right\|=m$ that $s^{R^{\prime}}$ is expressible in terms of the proposed basis elements. Also suppose that every $s^{R^{\prime \prime}}$ is expressed in terms of the proposed base elements if $\left\|R^{n}\right\|<m$. Let $R=\left(r_{1}, r_{2}, \ldots\right)$.

Case 1. If some $r_{2^{k}-1}=t \neq 0$ then

$$
S^{\left.t \Delta_{2} k-1 S^{\left(r_{1}, \ldots, r_{2} k\right.}-2,0, r_{2} k, \ldots\right)}=s^{R}+\text { larger terms. }
$$

Hence in this case $s^{R}$ can be suitably expressed.

Case 2. $r_{2^{k}}=t=4 r+\varepsilon$ where $\varepsilon=1,2$ or 3 . Taking the case $\varepsilon=3$ we have

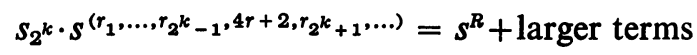

and $s^{R}$ is expressed in terms of the proposed base elements. Similarly if $\varepsilon=1$. 
Taking the case $\varepsilon=2$ we have

$$
S_{\left.2^{k}, 2^{k} S^{\left(r_{1}, \ldots, r_{2}{ }^{k}-1\right.}, 4 r, \ldots\right)}=S^{R}+\text { larger terms }
$$

and this case follows.

Case 3. $r_{2 k+1}=4 r+\varepsilon$ where $\varepsilon=1,2$ or 3. This goes exactly as Case 2 .

Case 4. $r_{2 k}=2 r+1, k \neq 2^{j}$.

$$
s_{2 k} s^{\left(r_{1}, \ldots, r_{2 k-1}, 2 r, \ldots\right)}=s^{R}+\text { larger terms. }
$$

Since $s_{2 k} \in U^{+}$, this case follows.

If none of the above hold, then $s^{R}$ is one of the proposed base elements. The theorem follows.

5. The main theorems. Recall that in $\S 2$ we have identified $\tilde{H}^{*}\left(M O ; Z_{2}\right)$ with the algebra $A^{\prime}=A \otimes Z_{2}$ of $\S 4$. If $N_{*}$ is the unoriented cobordism group, there is also the pairing $A^{\prime} \otimes N_{*} \rightarrow Z_{2}$ mapping $s^{R} \otimes[M]$ into $s^{R}[M]$. Letting $A^{\prime}=\sum A_{k}^{\prime}$ where $A_{k}^{\prime}$ is generated by all $s^{R}$ with $\|R\|=k$, we have

$$
A_{k}^{\prime} \otimes N_{k} \rightarrow Z_{2}
$$

Recalling that $\mathscr{S} \subset A^{\prime}$ is the Steenrod algebra, we know from Thom that $\left(\mathscr{S}^{+} \cdot A^{\prime}\right)_{k} \otimes N_{k} \rightarrow 0$ and that the induced map

$$
\left(A^{\prime} / \mathscr{S}^{+} \cdot A^{\prime}\right)_{k} \otimes N_{k} \rightarrow Z_{2}
$$

is a dual pairing.

Consider now the ring $T \supset \mathscr{S}$ of $\S 2$. Define $P_{k}$ as the subgroup of $N_{k}$ consisting of all $[M] \in N_{k}$ such that $\left(s_{2} \cdot s^{R}\right)[M]=0$ for all $s^{R}$ of degree $k-2$. Alternatively, $P_{k}$ is the annihilator of $\left(T^{+} \cdot A^{\prime}\right)_{k}$ in $A^{\prime} \otimes N_{k} \rightarrow Z_{2}$ and thus there is the dual pairing

$$
\left(A^{\prime} / T^{+} \cdot A^{\prime}\right)_{k} \otimes P_{k} \rightarrow Z_{2} \text {. }
$$

(5.2) $P=\sum P_{k}$ is a subalgebra of $N_{*}$.

Proof. If $[M],\left[M^{\prime}\right] \in P$, then

$$
\begin{aligned}
\left(s_{2} \cdot s^{R}\right)\left[M \times M^{\prime}\right]= & \sum_{R_{1}+R_{2}=R}\left(s_{2} s^{R_{1}}\right)[M] \cdot s^{R_{2}}\left[M^{\prime}\right] \\
& +\sum_{R_{1}+R_{2}=R} s^{R_{1}}[M] \cdot\left(s_{2} s^{R}\right)\left[M^{\prime}\right]
\end{aligned}
$$

and $\left[M \times M^{\prime}\right] \in P$.

(5.3) THEOREM. There exist generators $\left[M^{2}\right],\left[M^{4}\right],\left[M^{5}\right], \ldots$ for $N_{*}$ as a polynomial algebra over $Z_{2}$ such that $P$ is the polynomial subalgebra with generators $\left[M^{2}\right]^{2},\left[M^{4}\right]^{2},\left[M^{6}\right],\left[M^{8}\right]^{2},\left[M^{9}\right]^{2},\left[M^{10}\right], \ldots$ Here every $\left[M^{2 k+1}\right]$ and every $\left[M^{2^{k}}\right]$ occurs to the power two and every $\left[M^{2 k}\right]$ with $k \neq 2^{j}$ to the power one.

Proof. We know that $\left(A^{\prime} / T^{+} \cdot A^{\prime}\right)_{k}$ and $P_{k}$ are dually paired. In (4.3) the dimension of $\left(A^{\prime} / T^{+} \cdot A^{\prime}\right)_{k}$ has been obtained and it agrees with that of the above polynomial subalgebra. Hence we have only to show the existence of a polynomial 
basis for $N_{*}$ such that $\left[M^{2 k+1}\right]^{2},\left[M^{2^{k}}\right]^{2} \in P$ and $\left[M^{2 k}\right] \in P$ for $k \neq 2^{j}$. In the first cases the choice is arbitrary since

$$
\left(s_{2} s^{R}\right)[M \times M]=\sum s_{2} s^{R_{1}}[M] \cdot s^{R_{2}}[M]+\sum s^{R_{1}}[M] \cdot s_{2} s^{R_{2}}[M]=0 .
$$

Thus we have only to show the existence for $k \neq 2^{j}$ of an $\left[M^{2 k}\right] \in P_{2 k}$ with $s_{2 k}\left[M^{2 k}\right]$ $=1$. From (4.3) we know that $s_{2 k} \notin T^{+} \cdot A^{\prime}$ for $k \neq 2^{j}$. Since

$$
\left(A^{\prime} / T^{+} \cdot A^{\prime}\right)_{k} \otimes P_{k} \rightarrow Z_{2}
$$

is a dual pairing, there exists $\left[M^{2 k}\right] \in P_{2 k}$ with $s_{2 k}\left[M^{2 k}\right]=1$. The theorem follows.

(5.4) THEOREM. Define $Q_{k}$ to be the set of all cobordism classes [M] $\in N_{k}$ such that

(i) $\left(s_{2} \cdot s^{R}\right)[M]=0$, all $R$ with $\|R\|=k-2$, and

(ii) $\left(s_{2,2} \cdot s^{R}\right)[M]=0$, all $R$ with $\|R\|=k-4$.

Let $Q=\sum Q_{k}$. Then $Q$ is a subalgebra of $N_{*}$ and $Q=P^{2}=\left\{x^{2} \mid x \in P\right\}$.

Proof. It is readily checked that $Q$ is a subalgebra, and this is left to the reader. We have a dual pairing

$$
\left(A^{\prime} / U^{+} \cdot A^{\prime}\right)_{k} \otimes Q_{k} \rightarrow Z_{2}
$$

and an isomorphism

$$
\alpha: A^{\prime} / U^{+} \cdot A^{\prime} \approx A^{\prime} / T^{+} \cdot A^{\prime} .
$$

It is also readily checked that if $M \in P_{k}$ then $[M]^{2} \in Q_{2 k}$. The diagram

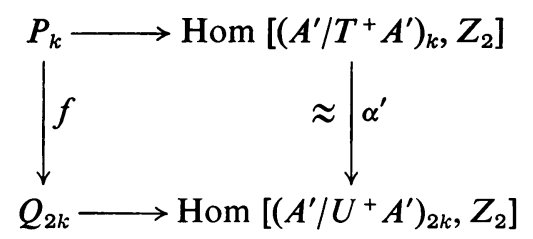

is commutative, where $f(x)=x^{2}$. Hence $f$ is an isomorphism and $Q_{2 k}=\left(P_{k}\right)^{2}$. The theorem follows.

It follows immediately from (5.3) that $P_{2 k+1}=0$. Hence in (5.4) we may replace (ii) by

(ii) $\left(s_{2,2} \cdot s^{R}\right)[M]=0$ when $\operatorname{dim} M=2 k,\|R\|=2 k-4$.

Let $E_{2 k}$ denote the subgroup of $N_{2 k}$ consisting of all cobordism classes represented by a weakly almost complex manifold $M^{2 k}$ such that its Chern numbers satisfy

$$
c_{2 r+1} c_{i_{1}} \cdots c_{i_{\gamma}}[M]=0, \quad r=0,1,2, \ldots
$$

Note that this condition is equivalent whether the stable tangent bundle or the normal bundle is used to define Chern classes. Let $E=\sum E_{2 k}$.

(5.5) THEOREM. The subalgebras $E, P$ and $\operatorname{Im}\left(\Omega_{*}^{S p} \rightarrow N_{*}\right)$ of $N_{*}$ satisfy $E \subset P^{4}$, $\operatorname{Im}\left(\Omega_{*}^{S p} \rightarrow N_{*}\right) \subset P^{8}$. 
Proof. The map $g: M S p \rightarrow M O$ has $g^{*}\left(s^{4 R}\right)={ }^{\prime} s^{R} \bmod 2, g^{*}\left(s^{R^{\prime}}\right)=0$ for $R^{\prime} \neq 4 R$. Hence for each $[M]$ in $\operatorname{Im}\left(\Omega_{*}^{S p} \rightarrow N_{*}\right)$ we have $s^{R^{\prime}}[M]=0$ for $R^{\prime} \neq 4 R$. From (3.6) and (3.7) we have

$$
\left(s^{4 \Delta_{2}} \cdot s^{R}\right)[M]=0, \quad\left(s^{8 \Delta_{2}} \cdot s^{R}\right)[M]=0,
$$

the second holding in case $\operatorname{dim} M=8 k$ and $\|R\|=8 k-16$.

From $s^{R^{\prime}}[M]=0$ unless $R^{\prime}=4 R$ it follows that $[M]=\left[M^{\prime}\right]^{4}$ for some $\left[M^{\prime}\right]$. For define $A^{\prime} \rightarrow Z_{2}$ by $s^{R} \rightarrow s^{4 R}[M]$. Alternatively there is $\alpha^{2}: A^{\prime} \rightarrow A^{\prime}$ and $\alpha^{2}(x) \rightarrow x[M]$, which is well defined. Then $\alpha^{2}\left(S q^{4 n} s^{4 R}\right)=S q^{n} \cdot s^{R}$, hence $S q^{n} \cdot s^{R} \rightarrow 0$ and the homomorphism $A^{\prime} \rightarrow Z_{2}$ kills $\mathscr{S}^{+} \cdot A^{\prime}$. Hence there is an [ $\left.M^{\prime}\right]$ with $s^{R}\left[M^{\prime}\right]=s^{4 R}[M]$, and $[M]=\left[M^{\prime}\right]^{4}$. Then

$$
\left(s^{\Delta_{2}} \cdot s^{R}\right)\left[M^{\prime}\right]=0, \quad\left(s^{2 \Delta_{2}} \cdot s^{R}\right)\left[M^{\prime}\right]=0,
$$

the latter holding in case $\operatorname{dim} M=8 k$. Then by (5.4) and the remark following it, we have $\left[M^{\prime}\right] \in P^{2}$ and $\operatorname{Im}\left(\Omega_{*}^{S p} \rightarrow N_{*}\right) \subset P^{8}$. In exactly the same way using (3.8) we see that $E \subset P^{4}$.

6. Remarks and questions. (a) It can be shown that every $[M] \in P_{k}$ is a Wall manifold, i.e.

$$
w_{1}^{2} w_{i_{1}} \cdots w_{i_{p}}[M]=0, \text { all } i_{1}, \ldots, i_{p} .
$$

Thus $P$ is contained in the Wall subalgebra $W$ of $N_{*}$. Clearly $P \neq W$ since $P_{5}=0$ and $W_{5} \neq 0$. It would be interesting to have a characterization of $P$ as a subalgebra of $W$. A generator for $W_{6}$ can also serve as generator for $P_{6}$. Hence [ $M^{6}$ ] can be taken to be the $C P(2)$ bundle over the Klein bottle used by Wall. I have not tried to obtain models for all the generators $M^{2 k}$.

(b) From $\$ 3$ one gets the impression that one might try to study divisibility relations between Chern numbers in terms of the algebra $A$. The advantage is that the Adams operations on $A \subset \widetilde{K}(M U)$, when suitably stabilized, can be put in terms of the multiplication of $A$. Can one understand the Stong results [9] on

$$
\tilde{K}(M U) \otimes \Omega_{*}^{U} \rightarrow Z
$$

purely arithmetically? Can one use $A$ to make a general arithmetic of divisibility relations?

(c) In (5.5) it has been shown only that $E \subset P^{4}$, but it seems safe to conjecture $E=P^{4}$. Since $P^{4}$ is a polynomial algebra with generators of dimensions $16,32,40,24,64,72,40, \ldots$, it would be enough to exhibit generators in $E$ in these dimensions. Generators in dimension $16,32,64, \ldots$ were constructed in $\$ 1$. By messy calculations I have shown the existence of an $M^{24}$. Hence in dimensions $k<40$ we have $E_{k}=\left(P^{4}\right)_{k}$. Are there polynomial generators of $P_{*}^{4}$ whose stable tangent bundles are complexifications of real bundles? Here the first unanswered dimension is 24 .

(d) $\operatorname{Is~} \operatorname{Im}\left(\Omega_{*}^{S p} \rightarrow N_{*}\right)=P^{8}$ ? 


\section{REFERENCES}

1. P. E. Conner and E. E. Floyd, Differentiable periodic maps, Ergebnisse der Math. und ihrer Grenzgebiete, Band 33, Academic Press, New York; Springer-Verlag, Berlin, 1964. MR 31 \#750.

2. - The relation of cobordism to K-theories, Lecture Notes in Math., no. 28, SpringerVerlag, Berlin and New York, 1966. MR 35 \#7344.

3. - Torsion in SU-bordism, Mem. Amer. Math. Soc. No. 60 (1966). MR 32 \#6471.

4. W. C. Hsiang and R. H. Szczarba, On the tangent bundle of a Grassmann manifold, Amer. J. Math. 86 (1964), 698-704. MR 30 \#2523.

5. P. S. Landweber, Cobordism operations and Hopf algebras, Trans. Amer. Math. Soc. 129 (1967), 94-110. MR 36 \#2145.

6. J. W. Milnor and J. C. Moore, On the structure of Hopf algebras, Ann. of Math. (2) 81 (1965), 211-264. MR 30 \#4259.

7. S. P. Novikov, The method of algebraic topology from the viewpoint of cobordism theories, Izv. Akad. Nauk SSSR Ser. Mat. 31 (1967), 855-951.= Math. USSR Izv. 1 (1967), 827-913. MR 36 \#4561.

8. David M. Segal, Symplectic cobordism of manifolds, Thesis, Massachusetts Institute of Technology, Cambridge, Mass., 1968; Comment. Math. Helv. 45 (1970), 159-169.

9. R. E. Strong, Relations among characteristic numbers. I, II, Topology 4 (1965), 267-281; Topology 5 (1966), 133-148. MR 33 \#740; MR 33 \#741.

10. — , Notes on cobordism theory, Princeton Univ. Press, Princeton, N. J.; Univ. of Tokyo Press, Tokyo, 1968.

11. - Some remarks on symplectic cobordism, Ann. of Math. (2) 86 (1967), 425-433. MR 36 \#2162.

12. R. Thom, Quelques propriétés globales des variétés différentiables, Comment. Math. Helv. 28 (1954), 17-86. MR 15, 890.

13. C. T. C. Wall, Determination of the cobordism ring, Ann. of Math (2) 72 (1960), 292-311. MR 22 \#11403.

14. Don D. Porter, Novikov resolutions for symplectic cobordism, Thesis, Northwestern University, Evanston, III., 1969.

UNIVERSITY OF VIRGINIA,

Charlottesville, Virginia 22903 